\title{
Influence of gravity on surface melting
}

\author{
H. Löwen and T. Beier \\ Sektion Physik der Universität München, Federal Republic of Germany
}

Received December 12, 1989

The influence of gravity on planar surface melting is studied if the surface normal is parallel to the gravitational acceleration. We find that gravity prevents complete melting. At the triple point, the solid is covered with a liquid film whose thickness is of the order of a micrometer. We suggest to use a centrifuge with high centripetal acceleration in order to observe the deviation from the usual melting law experimentally.

\section{Introduction}

Surface melting is an interesting current research subject, both experimentally and theoretically. Concerning recent reviews on this topic, we refer to van der Veen et al. [1] and Dash [2]. Extensive studies were made for lead where now the dependence on the orientation of the surface plane [3] and the role of the long range forces $[4,5]$ are well understood. In this case, one could approach the melting temperature up to the order of $m K$ ! In particular, the (110)-plane was shown to exhibit complete melting.

In the interpretation of the experimental data and also in current theories of surface melting, gravity is neglected. On the other hand, it is known from the Moldover-Cahn wetting experiments on three coexistent liquids [6] that gravity limits the width of the wetting layer [7]. It is thus tempting to examine the influence of gravity on surface melting.

In our chosen geometry the normal of the solid surface has the same direction as the gravitational acceleration [8]. Then our main result is that, at the triple point, the solid is covered with a liquid layer with a finite width $l^{*}$ and can even be overheated. $l^{*}$ is given by

$l^{*}=\left(2 W \rho_{S} /\left(m g \rho_{L}\left(\rho_{S}-\rho_{L}\right)\right)\right)^{1 / 4}$.

Here, $g$ is the acceleration, $m$ denotes the mass of a particle and $W$ the Hamaker constant of the material. Furthermore, $\rho_{S}$ and $\rho_{L}$ are the solid and liquid densities at the triple point. The triple gas density $\rho_{G}$ is assumed to be small

$\rho_{G} \ll \rho_{S}, \rho_{L}$

and we suppose

$\rho_{L}<\rho_{S}$.

For lead and for the gravity acceleration on the earth $\left(g \equiv g_{0}=9.81 \mathrm{~m} / \mathrm{s}^{2}\right): l^{*}=580 \mathrm{~nm}$, rather insensitive to $m, W$, etc. In typical experiments [4] one can approach widths of $l \leqq 8 \mathrm{~nm}$. That means that the deviations from complete melting due to gravity are nonobservable below the triple point. However, for very large $g$, $l^{*}$ is reduced. In a centrifuge, one can achieve up to $g=10^{6} \mathrm{~g}_{0}$. Then, the deviations from the usual complete melting law are accessible by experiments.

The physical reason of the finite width $l^{*}$ at the triple point is a competition in the free energy between interface repulsion, which drives complete melting and is for non-retarded van der Waals forces $\sim l^{-2}$ ( $l$ denoting the width of the liquid layer), and the contribution $\sim g l^{2}$ which arises from gravity and prevents melting for large $l$.

\section{Theory}

We start from the grand canonical energy functional $\Omega[\{\rho(\mathbf{r})\}, T, \mu]$ of the particle density $\rho(\mathbf{r})$ in an external potential $V(\mathbf{r})$ for a given temperature $T$ and chemical potential $\mu$. It may be written as $[9,10]$

$$
\begin{aligned}
\Omega[\{\rho(\mathbf{r})\}, T, \mu]= & \int \mathrm{d}^{3} r f_{h}[T,\{\rho(\mathbf{r})\}]+\int \mathrm{d}^{3} r(V(\mathbf{r})-\mu) \rho(\mathbf{r}) \\
& +(1 / 2) \int \mathrm{d}^{3} r \int \mathrm{d}^{3} r^{\prime} \tilde{w}\left(\left|\mathbf{r}-\mathbf{r}^{\prime}\right|\right) \rho(\mathbf{r}) \rho\left(\mathbf{r}^{\prime}\right) .
\end{aligned}
$$

Here, $f_{h}(T, \rho)$ is the free energy functional for a system with pure repulsive forces which we assume to be local. For small densities, $f_{h}(T, \rho)$ equals the ideal gas part

$f_{h}(T, \rho)=k_{B} T \rho\left(\ln \left(\Lambda^{3} \rho\right)-1\right)+\mathrm{O}\left(\rho^{2}\right)$. 
$A$ being the thermal wave length. Furthermore, $\tilde{w}(r)$ is the attractive part of the interparticle potential.

In what follows we specify the external potential to

$V(\mathbf{r}) \equiv V(z)= \begin{cases}\infty & z<0 \\ m g z & z \geqq 0\end{cases}$

As in (1), $m$ means the particle mass and $g$ the gravitational acceleration.

The occurrence of $V(z)$ implies that the system can be viewed as one with $z$-dependent chemical potential which may be called gravito-chemical potential

$\mu(z)=\mu-V(z)$.

We consider a process with a chemical potential $\mu$ $=\mu(T)=\mu_{0}+\mu_{S}(T)$ with $\mu(T) \gg \mu_{S}(T)$ where $\mu_{0}$ is fixed and $\mu_{S}(T)$ is the chemical potential on the sublimation line. Then, the gravito-chemical potential equals $\mu_{S}(T)$ at a macroscopic $T$-independent height (see Fig. 1)

$z_{0}=\mu_{0} / m g$

Then we raise the temperature $T$ towards the triple temperature $T_{T}$.

Concerning the density parameterization, we use a sharp kink approximation $[11,10]$ for the solid and liquid density $\rho_{S}, \rho_{L}$. As a crucial approximation we neglect the $\mu$-dependence of $\rho_{S}, \rho_{L}$. This should be a good approximation since the solid and liquid are nearly incompressible. It is merely for very high $g$ that the $\mu$-dependence of the solid and liquid density becomes essential, see Chap. 3. The vapor density is small and we assume that the vapor behaves like an ideal gas. Then, the total density contains two free paramters $l_{1}, l_{2}$ which determine the width of a liquid film between the solid and vapor phase, measured from $z_{0}$, see Fig. 1 .

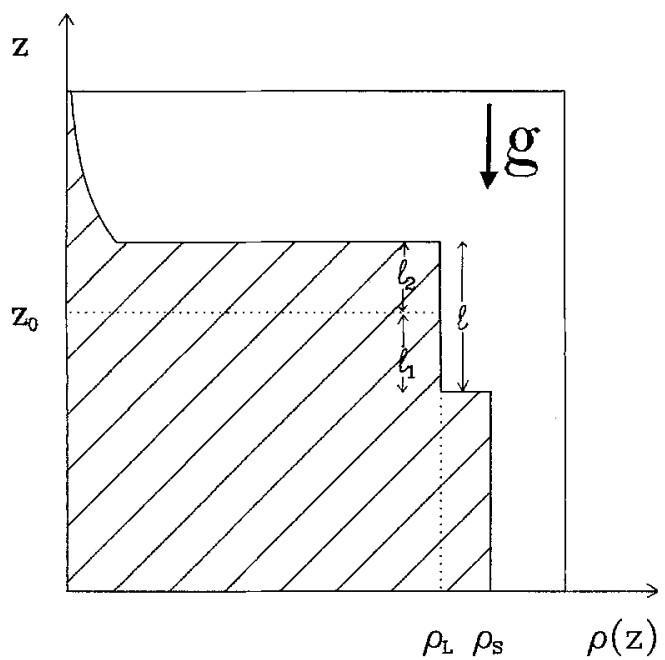

Fig. 1. Schematic plot of the density parameterization in a gravitational field: exponentially decreasing gas density, liquid layer with width $l$ and solid density in a sharp kink approximation
Summarizing, we assume

$$
\rho(z)= \begin{cases}0 & z \leqq 0 \\ \rho_{S} & 0<z \leqq z_{0}-l_{1} \\ \rho_{L} & z_{0}-l_{1}<z \leqq z_{0}+l_{2} \\ \rho_{G}(z) & z>z_{0}+l_{2}\end{cases}
$$

with the gas density

$\rho_{G}(z)=A^{-3} \exp \left((\mu-m g z) / k_{B} T\right)$.

Note that we assume (2), i.e. the gas density is small.

We insert the density $\rho(z)$ into the grand canonical free energy functional and minimize with respect to $l_{1}$ and $l_{2}$. It may be more convenient to use $l_{1}$ and the total width $l=l_{1}+l_{2}$ as variable. Then, minimization with respect to $l_{1}$ leads to

$l_{1}=\rho_{L} l / \rho_{S}$.

The remaining functional $\Omega$ is merely a function of $l$ and has to be minimized with respect to $l$. This function $\Omega$ per area $A$ reads as follows

$$
\begin{aligned}
& \Omega(l, T, \mu) / A=\omega_{S}\left(\rho_{S}, T, \mu_{S}(T)\right)\left(z_{0}+k_{B} T /(m g)\right) \\
& \quad-m g \rho_{S} z_{0}^{2} / 2+\sigma_{S, \text { vac }}+\sigma_{S, L}^{\mathrm{ex}}+\sigma_{L, G}^{\mathrm{ex}} \\
& \quad+l\left[\omega_{L}\left(\rho_{L}, T, \mu_{S}(T)\right)-\omega_{S}\left(\rho_{S}, T, \mu_{S}(T)\right)\right] \\
& \quad+\Sigma_{\mathrm{grav}}+\Sigma_{w}+\mathrm{O}\left(\left[m g\left(\rho_{S}-\rho_{L}\right) l /\left(k_{B} T \rho_{S}\right)\right]^{3}\right)
\end{aligned}
$$

with

$$
\begin{aligned}
& \Sigma_{\text {grav }}=m g \rho_{L}\left(\rho_{S}-\rho_{L}\right) l^{2} /\left(2 \rho_{S}\right), \\
& \Sigma_{w}=-\left(\rho_{S}-\rho_{L}\right) \rho_{L} \int_{i}^{\infty} \mathrm{d} y t(y) .
\end{aligned}
$$

Let us explain the notation: $\omega_{S}\left(\rho_{S}, T, \mu_{S}(T)\right)$ is the grand canonical free energy density of the bulk solid on the sublimation line. Near the triple temperature $T_{T}$, the difference of the (metastable) liquid and solid free energies equals

$\omega_{L}\left(\rho_{L}, T, \mu_{S}(T)\right)-\omega_{S}\left(\rho_{S}, T, \mu_{S}(T)\right) \cong L\left(T_{T}-T\right)$.

$L$ denoting a specific heat. Furthermore,

$t(y) \equiv \int_{y}^{\infty} \mathrm{d} x \int \mathrm{d}^{2} r \tilde{w}\left(\sqrt{r^{2}+x^{2}}\right)$.

Usually, the long ranged part of the particle interaction is of van der Waals type, proportional to $r^{-6}$. Hence

$\Sigma_{\text {grav }} \cong W / l^{2}$,

for large $l, W$ being the Hamaker constant. $\sigma_{S, \text { vac }}, \sigma_{S, L}^{\mathrm{ex}}$, $\sigma_{L, G}^{\mathrm{ex}} \cong \sigma_{L, \text { vac }}^{\mathrm{ex}}$ are the (extrapolated) surface tensions between solid/vacuum, solid/liquid and liquid/gas. Their explicit form is given in [9]. Finally, we remark that the corrections of third and higher order in $l$ are very small and can be neglected for micro- and mesoscopic widths. 


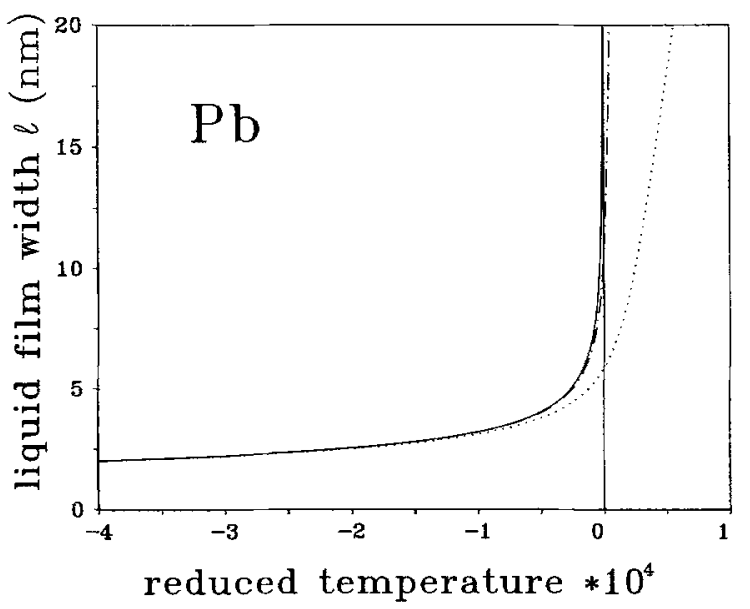

Fig. 2. Width of the liquid film versus reduced temperature $\left(T-T_{T}\right) / T_{T}$ for lead $\left(T_{T}=600.7 \mathrm{~K}\right)$ : The solid line corresponds to $g=0$ and is the usual $\left(T_{T}-T\right)^{1 / 3}$-law for complete surface melting. The dotdashed and dotted line are for $g=10^{8} \mathrm{~m} / \mathrm{s}^{2}, 10^{9} \mathrm{~m} / \mathrm{s}^{2}$ respectively. For $T=T_{T}, l$ is finite and the crystal is overheatable

Minimizing (12) with respect to $l$ and adopting (16)(17), we arrive at an implicit equation for $l=l(T)$ :

$L\left(T_{T}-T\right)=-m g \rho_{L}\left(\rho_{\mathrm{S}}-\rho_{L}\right) l / \rho_{L}+2 W / l^{3}$.

Discussing (18), we obtain that the width of the liquid layer remains finite for $T=T_{T}$ and even for $T>T_{T}$, i.e. the crystal can be overheated. For $T=T_{T}$, the width is given by (1). Using the material parameters for lead as given in [5], the function $l(T)$ is illustated in Fig. 2 for different accelerations $g$.

Let us finally comment on improvements of the theory and their influence on the result (18). First, one can use a better description of the reference free energy functional $\int \mathrm{d}^{3} r f_{h}(\{\rho(\mathbf{r}), T)$ in the spirit of a weighted density approximation [12]. Second, a more realistic density parameterization can be employed including Gaussian peaks for the solid density [13] and smooth interfaces [14] but still neglecting the $\mu$-dependence of the solid and liquid densities. These improvements merely lead to corrections of $l$ which are of the order of atomic distances and thus can be neglected if $l^{*}$ is large enough.

We add a remark to the peculiar case where the liquid is denser than the solid

$\rho_{L}>\rho_{\mathrm{S}}$

which is indeed realized e.g. for $\mathrm{Bi}, \mathrm{Si}, \mathrm{Ge}$. As is clearly seen from (14), (17), the Hamaker constant $W$ becomes negative in this case. It has been denoted by many authors [15] that this leads to blocked (incomplete) surface melting if $g=0$. However, with an external potential present the situation changes drastically. One has to recall that usually the liquid is thermodynamically stable for $T=T_{T}, \mu>\mu_{S}\left(T_{T}\right)$ if (19) is fulfilled. This means that the solid melts from its bottom for $T<T_{T}$. At the triple point two situations may occur. Usually

$\sigma_{L S}+\sigma_{S G}>\sigma_{L G}$ where $\sigma_{I J}$ means the surface tension between phases I and $J$ for $T=T_{T}$. In this situation there is no solid for $T=T_{T}$. In the opposite case

$\sigma_{L S}+\sigma_{S G}=\sigma_{L G}$

the solid covers the liquid phase. This is the same situation as for $\rho_{S}>\rho_{L}$, but now the roles of solid and liquid are interchanged.

\section{Discussion for large accelerations}

In conclusion, complete planar surface melting becomes incomplete if an external potential is present, and then the crystal is overheatable. This result is similar to the effect of finite size on surface melting $[16,17]$ where the melting can be incomplete, too. In the gravitational field of the earth, this effect is too small to be observable in experiment slightly below the triple temperature.

In order to observe this effect in experiments, we suggest to use a centrifuge where higher accelerations up to $10^{7} \mathrm{~m} / \mathrm{s}^{2}$ are available. In a centrifuge, the centrifugal potential is radially symmetric and reads as

$V(r)=-m \omega^{2} r^{2} / 2$

where $\omega$ is the angular velocity. If $R$ denotes the distance of the solid surface to the rotation axis, the potential $V(r)$ can be approximated linearly provided $R \gg l$ is valid where $l$ is the thickness of the liquid layer. The acceleration in a centrifuge is then given by

$g=R \omega^{2}$.

For very high $g$, the effect of gravity is dominant for $T \cong T_{T}$.

One remark concerning the density profile is in order. For small $g\left(g \lesssim 100 \mathrm{~m} / \mathrm{s}^{2}\right)$ the $\mu$-dependence of the solid and liquid densities is very weak and can be neglected as done in Sect. II. A sharp-kink approximation works well in this regime. If $g$ increases $\left(100 \mathrm{~m} / \mathrm{s}^{2}\right.$ $\lesssim g \lesssim 10^{4} \mathrm{~m} / \mathrm{s}^{2}$ ) the solid and liquid densities depend weakly on $\mu$ or equivalently on $z$. Nevertheless, since this dependence is weak, the result for $l^{*}$ (1) remains valid if one replaces $\rho_{L}$ and $\rho_{S}$ by mean densities of the interface region.

For very high $g\left(g \gtrsim 10^{4} \mathrm{~m} / \mathrm{s}^{2}\right)$ the real density profile $\rho(z)$ is not of sharp-kink type, but depends strongly on $z$, being a rather smooth decreasing function of $z$ with two interfaces. $\rho(z)$ will be the thermodynamically stable (metastable) solid (liquid) density for given $T$ and the gravitochemical potential $\mu(z)$. Since these densities are not known, in general, they cannot easily be incorporated into the effective interface potential and no general definitive prediction for $l^{*}$ can be made. Nonetheless we believe that the formula (1), (18) still make at least some approximate sense. We therefore take these formula to display the temperature dependence of the width of the quasiliquid layer for lead, even for very high accelerations, see Fig. 2. Here strong deviations from the usual complete melting behaviour are visible. Denoting these 
deviations by $\Delta l(T)=l(T)-l_{0}(T)$ where

$l_{0}(T)=\left(L\left(T_{T}-T\right) /(2 W)\right)^{-1 / 3}$

is the liquid film width without gravity for a temperature $T<T_{T}$, we find for small $\Delta l(T)$ :

$\Delta l(T) / l_{0}(T) \cong-\left(l_{0}(T) / l^{*}\right)^{4} / 3$

with $l^{*}$ given by (1). This implies that for $g=10^{7} \mathrm{~m} / \mathrm{s}^{2}$ which is available in a centrifuge, the deviations are large enough to be observable experimentally.

A second remark concerns the effect of a cylindrical shaped crystal surface which is enclosed in a centrifuge. Since $l \ll R$, the effect on surface melting is very small and negligible. More quantitatively, the leading term for wetting at the interior of a cylinder in the effective interface potential is [9]

$\Delta \Omega^{\mathrm{curv}}=\sigma_{L G} \cdot l / R$

which favors surface melting. Comparing with (12), this leads to a shift $\Delta t$ of the reduced temperature $t=\left(T-T_{T}\right) / T_{T}$. For lead, we have $\sigma_{L G}=0.501 \mathrm{~J} / \mathrm{m}^{2}[3]$ and for $R=1 \mathrm{~cm}$ we get $\Delta t \cong 10^{-8}$. The smallest resolution in $t$ which can be realized experimentally [5], however, is $\cong 10^{-6}$. Therefore it is justified to neglect the effect of cylindrical curvature completely.

Of course it is difficult to perform an experiment in a centrifuge, to keep the temperature constant etc., so it is a hard task to make exact measurements. The overheatability of the solid, however, is an effect which is even dominant for smaller accelerations; it should be much easier to detect it in a centrifuge experiment.

It is a pleasure to thank $H$. Wagner for useful discussions and helpful comments.

\section{References}

1. der Veen, J.F. van, Pluis, B., Denier, A.W. van der Gon: In: Chemistry and physics of solid surfaces. Vanselow, R., Howe, R.F. (eds.), p. 1701. Berlin, Heidelberg, New York: Springer 1988

2. Dash, J.G.: Contemp. Phys. 30, 89 (1989)

3. Pluis, B., Denier, A.W. van der Gon, Frenken, J.W.M., Veen, J.F. van der: Phys. Rev. Lett. 59, 2678 (1987)

4. Lipowsky, R.: Ferroelectrics 73, 69 (1987)

5. Pluis, B., Taylor, T.N., Frenkel, D., Veen, J.F. van der: Phys. Rev. B40, 1353 (1989)

6. Moldover, M., Cahn, J.: Science 207, 1073 (1980)

7. Gennes, P.G. de: J. Phys. (Paris) 42, L377 (1981)

8. Note that in the experiment of Ref. 5 the surface normal was perpendicular to gravity.

9. A review is: S. Dietrich. In: Phase transitions and critical phenomena. Domb, C., Lebowitz, J. (eds.), Vol. 12, p. 1. London: Academic Press

10. Dietrich, S., Schick, M.: Phys. Rev. B33, 4952 (1986)

11. Lipowsky, R., Kroll, D.: Phys. Rev. Lett 52, 2303 (1984)

12. Tarazona, P.: Phys. Rev. A31, 2672 (1985); Curtin, W.A., Ashcroft, N.W.: Phys. Rev. B32, 2909 (1985)

13. Dietrich S. (unpublished)

14. Napiorkowski, M., Dietrich, S.: Europhys. Lett. 9, 361 (1989)

15. see e.g.: Levi, A., Tosatti, E.: Surf. Sci. 189/190, 641 (1987); Löwen, H., Beier, T., Wagner, H.: Europhys. Lett. 9, 791 (1989); Z. Phys. B - Condensed Matter 79, 109 (1990)

16. Lipowsky, R., Gompper, G.: Phys. Rev. B29, 5213 (1984)

17. Löwen, H.: Equilibrium shapes of crystals near the triple point. Surf. Sci. (in press)

H. Löwen, T. Beier

Sektion Physik der Universität München

Theresienstrasse 37

D-8000 München 2

Federal Republic of Germany 